University of New Hampshire

University of New Hampshire Scholars' Repository

4-13-2005

\title{
An Estimate of the Gas Transfer Rate from Oceanic Bubbles Derived from Multibeam Sonar Observations of a Ship Wake
}

Thomas C. Weber

University of New Hampshire, Durham, thomas.weber@unh.edu

Anthony P. Lyons

Pennsylvania State University - Main Campus

David L. Bradley

Pennsylvania State University - Main Campus

Follow this and additional works at: https://scholars.unh.edu/ccom

Part of the Oceanography and Atmospheric Sciences and Meteorology Commons

\section{Recommended Citation}

Weber, T. C., A. P. Lyons, and D. L. Bradley (2005), An estimate of the gas transfer rate from oceanic bubbles derived from multibeam sonar observations of a ship wake, J. Geophys. Res., 110, C04005, doi:10.1029/2004JC002666.

This Journal Article is brought to you for free and open access by the Center for Coastal and Ocean Mapping at University of New Hampshire Scholars' Repository. It has been accepted for inclusion in Center for Coastal and Ocean Mapping by an authorized administrator of University of New Hampshire Scholars' Repository. For more information, please contact Scholarly.Communication@unh.edu. 


\title{
An estimate of the gas transfer rate from oceanic bubbles derived from multibeam sonar observations of a ship wake
}

\author{
Thomas C. Weber, Anthony P. Lyons, and David L. Bradley \\ Applied Research Laboratory, Pennsylvania State University, State College, Pennsylvania, USA \\ Received 13 August 2004; revised 21 December 2004; accepted 21 February 2005; published 13 April 2005.
}

[1] Measurements of gas transfer rates from bubbles have been made in the laboratory, but these are difficult to extrapolate to oceanic bubbles where populations of surfactants and particulate matter that inhibit gas transfer are different. Measurements at sea are complicated by unknown bubble creation rates that make it difficult to uniquely identify and observe the evolution of individual bubble clouds. One method that eliminates these difficulties is to measure bubbles in a ship wake where bubble creation at any given location is confined to the duration of the passing ship. This method assumes that the mechanisms slowing the gas dissolution of naturally created bubbles act in a similar manner to slow the dissolution of bubbles in a ship wake. A measurement of the gas transfer rate for oceanic bubbles using this method is reported here. A high-frequency upward-looking multibeam echosounder was used to measure the spatial distribution of bubbles in the wake of a twin screw 61-m research vessel. Hydrodynamic forcing functions are extracted from the multibeam data and used in a bubble cloud evolution model in which the gas transfer rate is treated as a free parameter. The output of model runs corresponding to different gas transfer rates is compared to the time-dependent wake depth observed in the data. Results indicating agreement between the model and the data show that the gas transfer rate must be approximately 15 times less then it would be for surfactant-free bubbles in order to explain the bubble persistence in the wake.

Citation: Weber, T. C., A. P. Lyons, and D. L. Bradley (2005), An estimate of the gas transfer rate from oceanic bubbles derived from multibeam sonar observations of a ship wake, J. Geophys. Res., 110, C04005, doi:10.1029/2004JC002666.

\section{Introduction}

[2] An understanding of the processes affecting the lifetime of bubbles is important for the remote sensing of ships through the use of acoustic techniques or synthetic aperture radar (SAR) as well as the study of air-sea interaction and its effect on the Earth's climate. After creation, bubbles undergo a complex evolution that is controlled by gas diffusion, turbulent diffusion, buoyant convection, and advection by local currents. During this time they scavenge surfactants and particulate matter from the surface waters in which they reside, and this can slow the gas dissolution process and significantly prolong their lifetime [Turner, 1961; Johnson and Cooke, 1981; Johnson and Wangersky, 1987; Blanchard, 1989]. As bubbles reach the surface and burst they eject both film and jet drops that are then mixed up into the air, releasing aerosols, scavenged bacteria, and other organic material [Monahan, 1986; Blanchard, 1989; Spiel, 1998]. Scavenged material collected at the surface reduces surface tension, resulting in a wake "scar" that is detectable by SAR for tens of km [Reed et al., 1990; Peltzer et al., 1992]. Bubbles also make a significant contribution to the exchange of gas between the

Copyright 2005 by the American Geophysical Union. 0148-0227/05/2004JC002666 atmosphere and the ocean [Woolf and Thorpe, 1991; Farmer et al., 1993; Woolf, 1997].

[3] Bubble persistence and the scavenging of materials are intertwined in a complex fashion: Bubbles that have longer lifetimes have more opportunity to scavenge material and a greater likelihood of reaching the surface before dissolving, while at the same time the scavenged material impedes gas transfer out of the bubble. Very small bubbles (less then $7 \mu \mathrm{m}$ in radius) were stabilized by surfactant films in filtered seawater by Johnson and Cooke [1981] and observed to last for several hours. Larger bubbles stabilized by adsorbed particles have been shown to last for weeks in a static laboratory setting, although the introduction of dynamic pressure changes that would be experienced by bubbles in a more natural environment was observed to destabilize some bubbles [Johnson and Wangersky, 1987]. The rate at which gas diffuses out of the bubbles is likely to depend on the nature of the surfactants and particles (i.e., size and type) and, as Johnson and Cooke [1981] suggest, on environmental conditions such as sea state, season, and atmospheric pressure.

[4] Quantitative measurements of gas transfer rates from bubbles have been made in the laboratory by Motarjemi and Jameson [1978] and Bischof et al. [1991], but these are difficult to extrapolate to oceanic bubbles where surfactant and particulate populations are different from the distilled, tap, or sewage water used in these experiments. In at-sea 


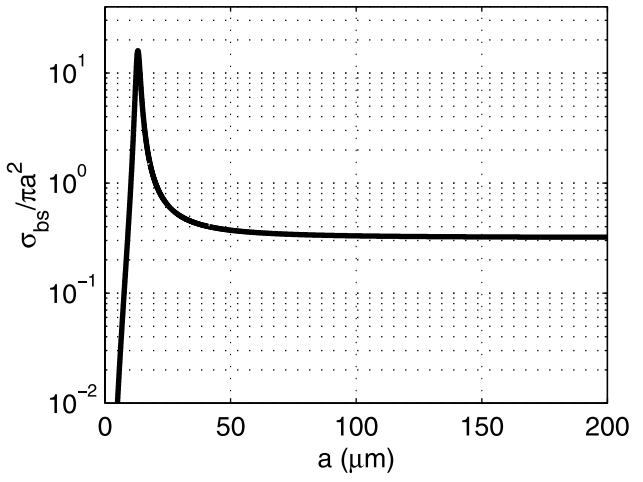

Figure 1. Ratio of the backscattering cross section to the geometric cross section for a single bubble at an incident frequency of $240 \mathrm{kHz}$ and at a depth of $1 \mathrm{~m}$.

experiments it is difficult to isolate gas transfer rates due to bubbles from other mechanisms, although measurements indicate that bubbles play a significant role [Wallace and Wirick, 1992; Farmer et al., 1993; Nakayama et al., 2000]. One possible method for examining gas transfer due exclusively to bubbles is to measure the persistence of a bubble cloud and then infer the gas transfer rate that is required to explain this persistence. Such measurements are difficult to make under breaking waves where new bubbles are being created and mixed downward into the older bubble cloud, but are feasible for bubbles created in a ship wake where bubble generation at a particular location is confined to the duration of the passing ship. Although the ship-induced hydrodynamic forcing functions that mix the bubbles downward into the water are different than ambient forcing functions, the surfactants and particulate matter that inhibit gas dissolution are expected to be the same.

[5] Measurements of bubble clouds are often made using acoustic remote sensing techniques that exploit the high scattering cross section of bubbles due to their resonant behavior [Clay and Medwin, 1977], although relatively few acoustic measurements of bubbles in ship wakes have been reported in the literature. The most extensive set of measurements were made by Trevorrow et al. [1994] from a suite of active sonars looking upward in the water column. These measurements were made at multiple frequencies, making it possible to observe the behavior of different size bubbles. Trevorrow et al. made measurements from three ships with lengths between $40 \mathrm{~m}$ and $65 \mathrm{~m}$ that had acoustically observable wakes lasting approximately 7-8 min. Multifrequency measurements were also made by Pidsley [1992], although they were reported much less extensively. Ship wake measurements have also been made with horizontally profiling ADCPs [Pinkel et al., 1995; Marmorino and Trump, 1996], and with a highresolution single-frequency $(68 \mathrm{kHz})$ multibeam sonar towed at a fixed distance by Gallaudet [2001], that showed evidence of twin vortices shed from the ship's hull moving in a transverse direction to that of the ship. This phenomenon has also been suggested by modeling [Reed et al., 1990; Hyman et al., 1995] and SAR measurements [Lyden et al., 1988], and is important because such vortices can create convergence zones in regions where they oppose the buoyant rise of the bubble. This is an important consideration when examining bubble persistence.

[6] The purpose of this paper is to report an estimate of the gas transfer rate that was inferred from high-frequency $(240 \mathrm{kHz})$ upward-looking multibeam echosounder data of the wake of a twin-screw 61-m research vessel. Benign weather conditions and low current speeds made it possible to collect wake profiles every $10 \mathrm{~s}$ or so for the entire lifetime of the wake. The data indicate strong evidence of the twin vortex structure mentioned above, and information regarding the strength of these vortices was extracted and used in a model describing the evolution of the bubbles in the wake. The bubble model, which is based on that used by Leifer and Patro [2002], treats the gas transfer rate as a free parameter. Model runs corresponding to different gas transfer rates are compared to wake features observed in the data. Of particular use in comparing model to data is the wake depth as a function of time or, equivalently, distance astern of the ship. As might be expected, the match between the model and the data indicates that the gas transfer rate is significantly longer than that predicted for a clean, surfactant-free bubble. It is important to note, however, that the specific gas transfer rates found here may apply only to the specific geographic location and time of year for which the measurements were conducted.

\section{Acoustic Backscatter From Bubbles}

[7] Acoustic backscatter in the ocean is often dominated by scattering from bubbles when they are present due to resonance effects. For a single bubble, the backscattering cross section $\sigma_{b s}$ is [Clay and Medwin, 1977]

$$
\sigma_{b s}(a)=\frac{a^{2}}{\left[\left(f_{r} / f\right)^{2}-1\right]^{2}+\delta^{2}},
$$

where $a$ is the bubble radius and $f$ is the acoustic frequency exciting the bubble. The resonant frequency $f_{r}$ is approximately (neglecting surface tension and assuming that there is no heat transfer between the bubble and the surrounding water as it oscillates)

$$
f_{r}(a)=\frac{1}{2 \pi a} \sqrt{\frac{3 \gamma P_{o}}{\rho_{w}}},
$$

where $\gamma$ is the ratio of specific heats for the gas inside the bubble, and $P_{o}$ and $\rho_{w}$ are the ambient pressure and density outside the bubble. A single bubble's backscattering cross section can be orders of magnitude higher then its geometric cross section. The $Q$ or sharpness of the amplitude response with respect to frequency near resonance is governed by the damping constant $\delta$ which includes contributions from shear viscosity, thermal conductivity, and reradiation. As the bubble size decreases, the contributions from both viscous damping and thermal conductivity increase [Clay and Medwin, 1977], and the $Q$ is lowered. The backscattering cross section normalized by the geometric cross section for bubbles with radii between $1 \mu \mathrm{m}$ and $200 \mu \mathrm{m}$ at an incident frequency of $240 \mathrm{kHz}$ and for a depth of $1 \mathrm{~m}$ is shown in Figure 1, where the resonant frequency and the damping 


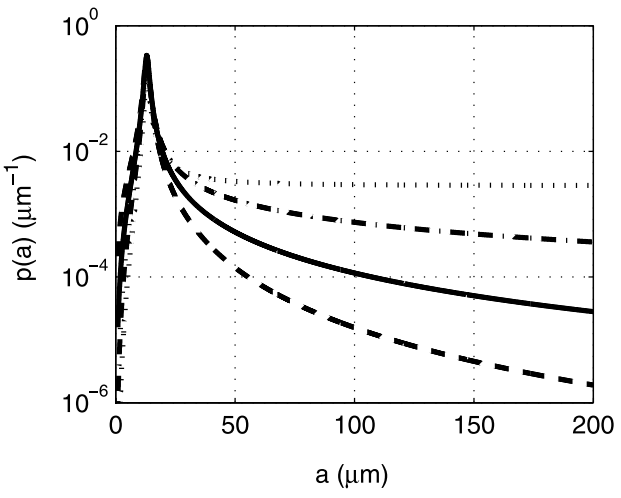

Figure 2. Relative contributions of different bubble sizes to backscatter at $240 \mathrm{kHz}$ for a cloud of bubbles whose distribution varies as $\mathrm{a}^{-\mathrm{m}}$ where $\mathrm{m}$ takes on values of 2 (dotted line), 3 (dash-dotted line), 4 (solid line), and 5 (dashed line).

constant both include contributions from shear viscosity and thermal conductivity following Clay and Medwin [1977]. A clear resonance is observed in Figure 1 where the backscattering cross section is 16 times larger then the geometric cross section. However, single bubbles that are significantly above resonance have backscattering cross sections that are approximately one third of their geometric cross sections and act as greater scatterers of sound. The contributions from bubbles below resonance are generally negligible.

[8] The backscatter from a unit volume containing a cloud of bubbles $s_{v}$ can be defined as

$$
s_{v}=\int \sigma_{b s}(a) n(a) d a
$$

where the size dependency of the backscattering cross section for individual bubbles is modulated by the bubble size distribution $n(a)$ which describes the average number of bubbles within the unit volume between a radius of $a$ and $a+d a$. The bubble size distribution in oceanic bubble plumes can vary greatly, but has often been observed to be approximately proportional to $a^{-4}$ [e.g., $W u, 1981$; Vagle and Farmer, 1992]. Backscatter from this type of bubble plume will be dominated by resonant bubbles even though the back scattering cross section is increasing as $a^{2}$ for bubbles above resonance. Dahl [2001] suggested a useful metric for evaluating the relative contributions of a distribution of bubble sizes to backscatter at a single frequency by defining the following density function, $p\left(a_{s}\right)$,

$$
p\left(a_{s}\right)=\frac{\sigma_{b s}\left(a_{s}\right) n\left(a_{s}\right)}{\int \sigma_{b s}\left(a_{s}\right) n\left(a_{s}\right) d a_{s}},
$$

where $a_{s}$ is the radius of the bubble contributing to the backscatter. This quantity is plotted in Figure 2 at $240 \mathrm{kHz}$ for four different bubble size distributions which vary as $a^{-m}$ where the exponent $m$ takes on values of $2,3,4$, and 5 , and shows that bubbles near resonance will dominate the integrated backscattering cross section. It should be pointed out, however, that if the slope of the bubble size distribution rolls off with decreasing bubble size before resonance, as was the case for Dahl [2001], off-resonance scattering can be significant.

\section{Multibeam Measurements of the Bubbly Wake}

[9] Measurements of the wake were made with a Reson 8101 multibeam echosounder. This sonar operates at a center frequency of $240 \mathrm{kHz}$ and transmits short pulses from a line array with a beam pattern that is nominally $1.5^{\circ} \times 170^{\circ}$. Backscattered acoustic signals are received on 101 identical $1.5^{\circ} \times 1.5^{\circ}$ beams that are oriented to make measurements in a $150^{\circ}$ sector of a plane. The multibeam sonar head was mounted on a subsurface buoy in a threepoint mooring, and was oriented so that the swath of beams was $30^{\circ}$ below the vertical (see Figure $3 \mathrm{a}$ ). This geometry was chosen so that the specular (mirror-like) surface reflections on the beams near nadir would not cause sidelobe interference on the adjacent beams. Data collected by the sonar head were transmitted through a fiber optic cable to a processor aboard a support ship approximately $600 \mathrm{~m}$ away (Figure 3b). The beam time series calculated by the processor were recorded on a PC. Owing to the slow data transmission speed between the sonar processor and the PC, wake images (i.e., the complete time series for all 101 beams) were only recorded approximately every $10 \mathrm{~s}$.

[10] The wake was created by the $\mathrm{M} / \mathrm{V}$ Indianapolis, which is $61 \mathrm{~m}$ long and has a draft of approximately $4.1 \mathrm{~m}$. (a)

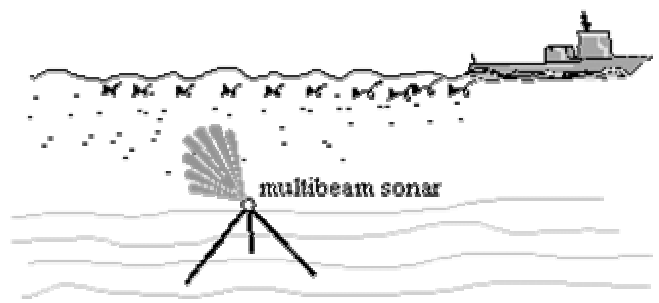

(b)

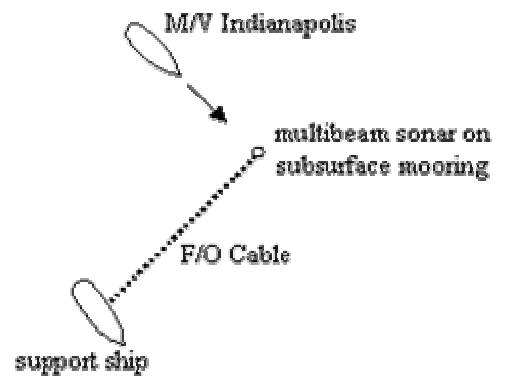

Figure 3. Experimental setup. (a) The multibeam sonar mounted in a three-point mooring $30 \mathrm{~m}$ below the sea surface. (b) The geometry describing the positions of the multibeam, the support vessel, and the $\mathrm{M} / \mathrm{V}$ Indianapolis that laid the wake. 


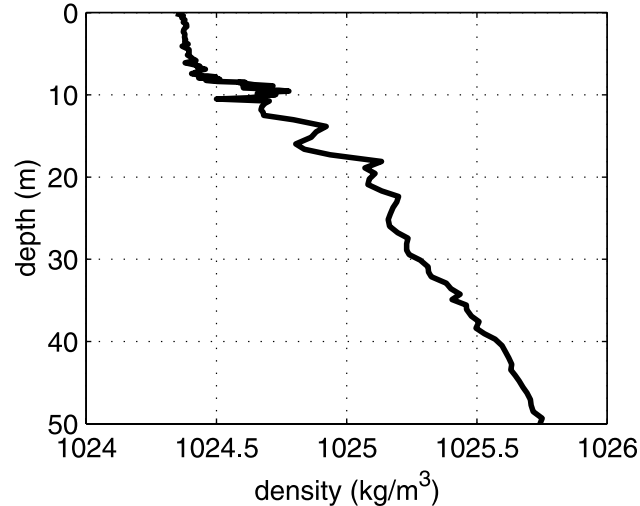

Figure 4. A density profile estimated from a CTD cast on the day of the experiment.

The Indianapolis has twin screws with four fixed 2.1-m blades $\left(71.5^{\circ} \mathrm{pitch}\right)$ that are counter-rotating (looking at the stern toward the bow, the port screw turns clockwise and the starboard screw turns counterclockwise). For the data described herein both shafts were turning at $288 \mathrm{rpm}$ with a corresponding vessel speed of $6.425 \mathrm{~m} / \mathrm{s}$. The environmental conditions at the time of these measurements were benign. The local surface currents were less then $0.1 \mathrm{~m} / \mathrm{s}$, the significant waveheight was slightly less then $0.25 \mathrm{~m}$, and the wind speed was minimal. CTD casts were taken during the experiment and showed a mixed layer depth of approximately $7.5 \mathrm{~m}$ (Figure 4). The temperature in the mixed layer was measured to be $20.1{ }^{\circ} \mathrm{C}$. The measurements were taken during August of 2002 off the coast of southern California.

[11] The raw data output from the multibeam sonar processor consists of the 101 beam time series, each of which is sampled at $15 \mathrm{kHz}$. A complete set of beam time series are collected approximately every $10 \mathrm{~s}$ and converted to volumetric scattering strength $S_{v}$ using a standard sonar equation given here in decibels [see Urick, 1983],

$$
S_{v}=\mathrm{RL}-\mathrm{SL}+2 \mathrm{TL}-10 \log _{10} V,
$$

where $\mathrm{RL}$ is the receive level at the multibeam hydrophone array, SL is the source level, TL describes the one-way transmission loss, and $V$ is the range-dependent ensonified volume calculated using the $1.5^{\circ} \times 1.5^{\circ}$ beamwidth and the $225 \mu$ s pulse length. Both the receive level and the source level are calculated based on a previous calibration of this sonar system [Weber, 2004]. Also, it should be noted that the transmission loss does not include the attenuation due to bubbles, which is not possible to calculate without an estimate of the bubble size distribution. Commonly used techniques to estimate the bubble size distribution use multifrequency acoustic techniques [e.g., Vagle and Farmer, 1998] which were not a component of this experiment. This means that estimates of scattering strength within the bubble plumes will be underestimated: Attenuation due to bubbles can be several dB/m [Trevorrow et al., 1994].

[12] An example of a multibeam wake image is shown in Figure 5a. There are several important features of this data

(a)

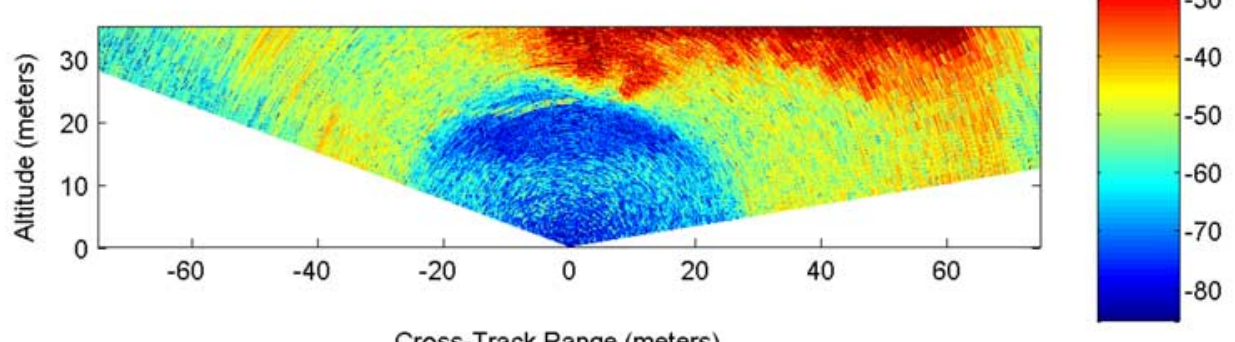

Cross-Track Range (meters)

(b)

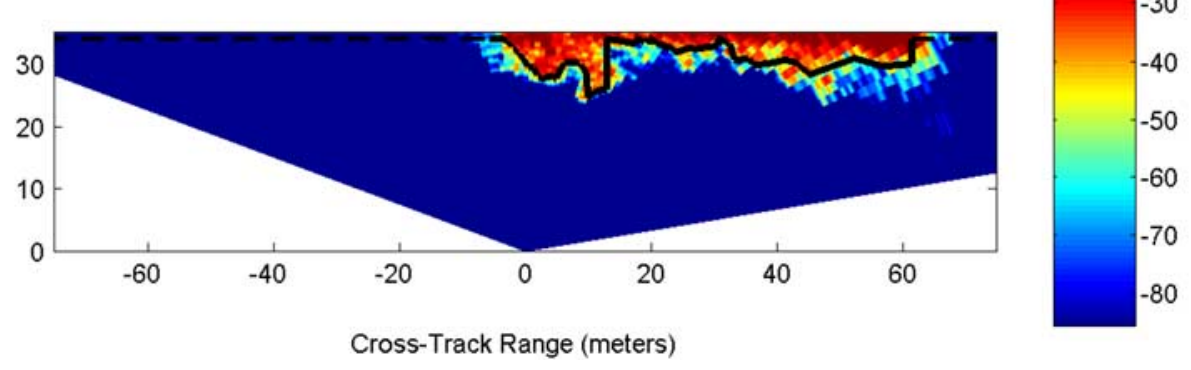

Figure 5. Multibeam wake data. (a) Unprocessed volumetric scattering strength data in $\mathrm{dB}$. The sonar is located at $(0,0)$ looking upward at the surface which appears at an altitude of $35 \mathrm{~m}$. (b) Filtered and thresholded volumetric scattering strength data with the overlaid wake profile (solid line) and the location of the sea surface (dashed line). 
(a)

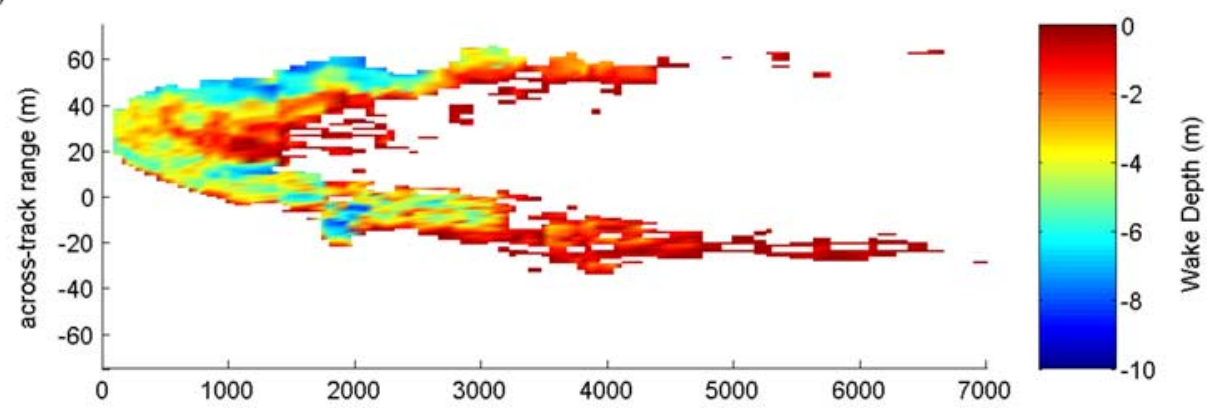

(b)

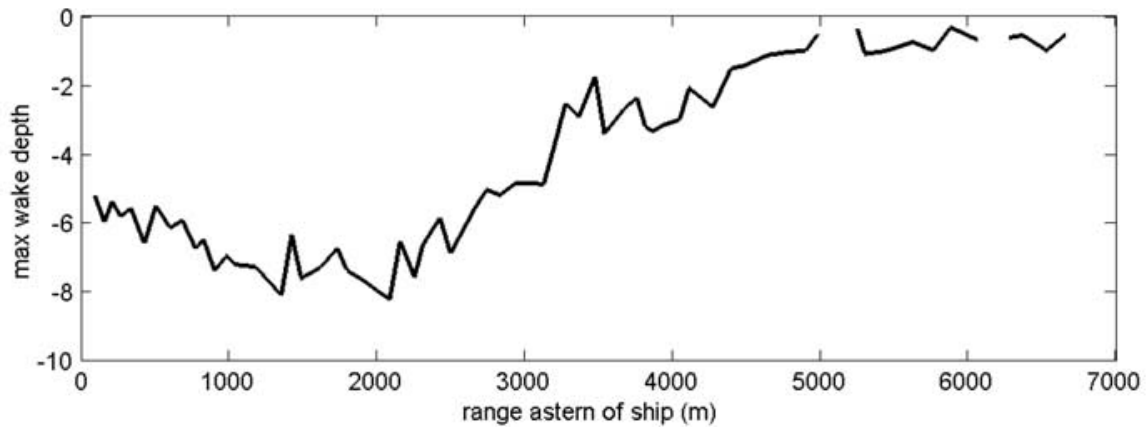

Figure 6. Wake of the $\mathrm{M} / \mathrm{V}$ Indianapolis. (a) The three-dimensional wake profile with color corresponding to depth. (b) The maximum depth (averaged between the port and starboard wake components) as a function of range astern of the ship.

set. The first is the minimal surface return where there is no bubble plume, which is an indicator of how calm and flat the sea surface was. Sidelobe interference from the bubble plume is visible as depicted by the yellow band at the ranges corresponding to the bubble plume. This places a limit on the dynamic range of this system, making it difficult to measure low-density bubble plumes near regions of high backscattering intensity. The sonar was tilted 30 degrees from the vertical to minimize sidelobe interference from specular surface returns.

[13] The profile of the wake was calculated from the volumetric scattering strength data by first filtering and thresholding the data and then amplitude detecting the first return before the known surface range in each beam. A lowpass spatial filter with a corner frequency of $\sim 0.35 \mathrm{~m}^{-1}$ was used along each beam. It would have been more appropriate to filter each data point (i.e., a given beam number and range) in time, but the slow data rate made this impossible. The choice to use spatial filtering instead is equivalent to an assumption of local (in time) ergodicity. A threshold value of $-37.5 \mathrm{~dB}$ was determined by examining which values best characterized the wake boundary in the region where the gradient in the scattering cross section was the highest. A typical result of the filtering and thresholding operation is shown in Figure $5 \mathrm{~b}$ with the wake profile overlaid on the filtered, thresholded data. Although some small portion of the wake is visible outside of the boundary, the most dense portions are generally well within it.

[14] Three-dimensional wake profiles were constructed by processing sequences of wake images and retaining each two-dimensional wake profile (i.e., the solid black line in Figure 5b). An example of the three-dimensional wake profile from the $\mathrm{M} / \mathrm{V}$ Indianapolis is shown in Figure 6a, where the time at which the data for the two-dimensional profiles was collected has been converted to distance astern of the ship using the ships speed and the assumption that the wake is not drifting. The maximum depth of the wake as function of distance astern of the ship is shown in Figure $6 \mathrm{~b}$. The wake reaches depths greater then $8 \mathrm{~m}$ at distances of more then $2 \mathrm{~km}$ astern of the ship. The subsurface expression of the wake is visible as far as $6 \mathrm{~km}$ astern of the ship. The persistence of the bubbles in the wake observed here, which are detectable below the surface for longer then 15 min, is also not unusual. It was known as early as WWII that bubbles can persist in a wake for up to $10 \mathrm{~min}$ [National Defense Research Committee, 1946]. More recent measurements by SAR show that wakes can exhibit dark centerlines for tens of kilometers astern of the ship [Reed et al., 1990; Peltzer et al., 1992]. This is attributed to the process of bubble scavenging which brings surfactants to the ocean surface where they reduce the surface tension and damp both capillary and small surface-gravity waves [Reed et al., 1990]. This suppression of surface roughness decreases the radar scattering cross section, causing the dark lines in the SAR images.

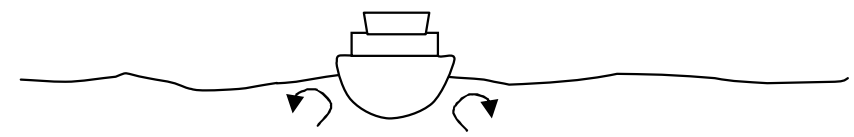

Figure 7. A cartoon depicting twin vortices being shed from the hull of the ship. Image vortices above the surface required to satisfy the surface boundary condition would cause the vortices to propagate away from the ship's track. 
(a)

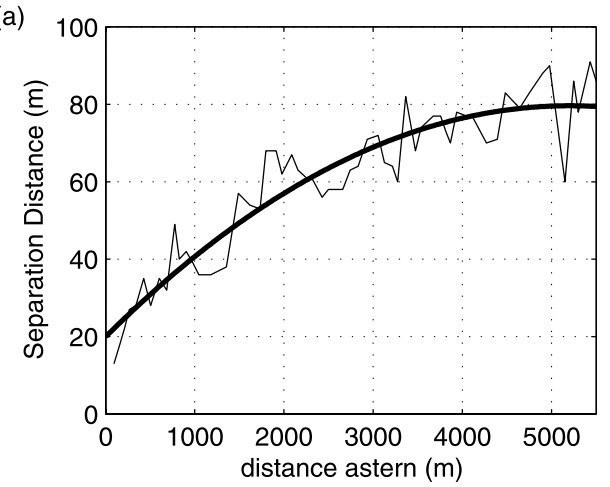

(b)

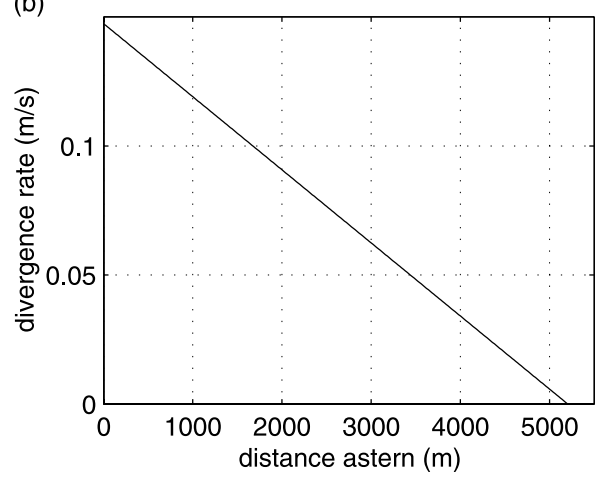

Figure 8. (a) The observed distance between the deepest wake components on each side of the ship's track with a second-order polynomial fit overlaid. (b) The separation rate of the vortices as a function of distance astern of the ship.

[15] The most striking feature in these data is that the wake splits into two distinct "tails" less then $1 \mathrm{~km}$ astern of the ship. At $1.5 \mathrm{~km}$ astern of the ship the wake is completely separated into port and starboard components (this corresponds to slightly more then $5 \mathrm{~min}$ after the ship has passed over the top of the multibeam sonar). The port and starboard wake components drift apart during the lifetime of the wake at a slowly decreasing speed. This feature is thought to be the manifestation of twin vortices shed by the displacement of the ship's hull as it passes through the water (Figure 7). These vortices propagate away from the ship's track in opposite directions entraining bubbles along the way, and it is the entrained bubbles that are observed by the multibeam sonar. Bubbles that are not entrained by the twin vortices presumably rise to the surface or dissolve due to gas diffusion.

[16] The strength of the vortices can be calculated by the rate at which the vortices are diverging from the ship's track [Kundu and Cohen, 2002]. In order to satisfy a flat surface boundary condition, each vortex will have an image vortex reflected above the surface. It is assumed that each vortex is axisymmetric with a 0.25 -m-diameter core that is in solid body rotation surrounded by an irrotational flow with a velocity that is inversely proportional to the distance from the vortex center. The angular component of the velocity outside of the core, $v_{\theta}$, can be written as

$$
v_{\theta}=\frac{\Gamma}{2 \pi r},
$$

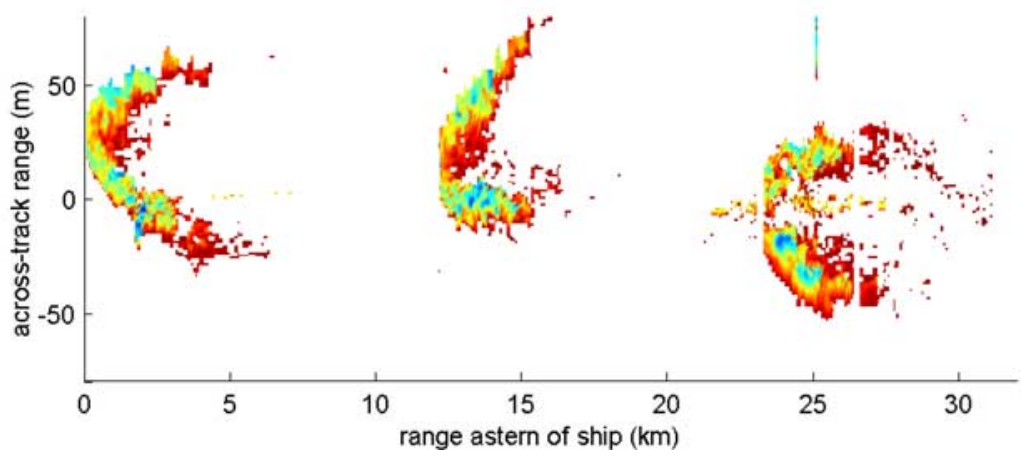

where $\Gamma$ is the circulation and $r$ is the radial distance from the vortex center. For the real vortex and its image vortex that are separated by a distance $h$, which is equal to twice the depth of the vortex center, the circulation is proportional to the speed at which the vortices are translating,

$$
\Gamma=2 \pi h v_{s} .
$$

For the ship wake described here $v_{s}$ the speed at which the vortices are translating away from the ships track, and the vortex model parameterization is completed by estimating the rate of separation of the two wake components and the depth of the real vortex center. It is assumed here that the vortex center occurs at $3 \mathrm{~m}$ depth (slightly more then half the ship's draft). The rate of separation is found by fitting a second-order polynomial to the range-dependent separation distance between the two wake components in order to estimate the initial rate of separation and the rate at which the vortex is slowing down (Figure 8). This results in an initial vortex separation rate of $0.147 \mathrm{~m} / \mathrm{s}$ and a deceleration of $1.82 \times 10^{-4} \mathrm{~m} / \mathrm{s}^{2}$.

[17] Another interesting feature of this data set is the bias of the wake toward one side, which is more apparent in the three successive wake images shown in Figure 9. The wake described in Figure 6 corresponds to the first wake that appears in Figure 9, and again both the three-dimensional wake profiles and the surface scattering strength are shown. The three wakes were generated within about 1.5 hours. The first wake corresponds to a heading of $150^{\circ}$, after which the

Figure 9. Wakes from three successive passes of the $\mathrm{M} / \mathrm{V}$ Indianapolis. 
Indianapolis turned around and ran a reciprocal course at $330^{\circ}$, and finally repeated the first run at a heading of $150^{\circ}$. This bias in the wake always corresponds to a particular side of the ship and so is not thought to be associated with an environmental effect such as drift due to local currents. It is also not thought to be associated with vortex shedding from the propellers because they are counter-rotating and turning at approximately identical shaft speeds. One possibility is that this effect is caused by an offset between the ship's yaw and true heading (i.e., the ship is "crabbing" through the water).

\section{Bubble Evolution Model}

[18] A model of the bubbles within the wake of the Indianapolis has been used in order to examine the hypothesis that large, slow-moving vortices are responsible for the wake separation and to examine the issue of bubble persistence in the wake with regard to a predicted gas dissolution rate. Several bubble evolution models have been previously described in the geophysical literature [e.g., Miner and Griffen, 1987; Thorpe et al., 1992; Woolf and Thorpe, 1991; Leifer and Patro, 2002]. Such models are typically Lagrangian tracers of individual bubbles that incorporate the effects of turbulent diffusion, gas diffusion, buoyancy, and sometimes other hydrodynamic features such as Langmuir circulation. These models are difficult to construct owing to incomplete knowledge about the complex processes involved (particularly turbulence and gas diffusion rates), but can be very useful in examining general trends and the relative importance of various phenomenon, as well as sensitivity studies for improving experimental measurements. It should also be pointed out that models describing the evolution of bubbles can be found in the chemical engineering literature where gas transfer from bubbles is important for many processing including oxygenation and the transfer of reactants [e.g., Ponoth and McLaughlin, 1999].

[19] The model used in this work is a Lagrangian bubble tracking model based on the one described by Leifer and Patro [2002], which starts with time-differentiating the ideal gas law, $P V=N R T$, and arranging the result to find the time rate of change of the bubble radius, $a$,

$$
\frac{\partial a}{\partial t}=\frac{R T\left[\frac{\partial N_{O 2}}{\partial t}+\frac{\partial N_{N 2}}{\partial t}\right]-\frac{4}{3} \pi a^{3} \rho_{w} g \frac{\partial z}{\partial t}}{4 \pi a^{2}\left(P_{A}+\rho_{w} g z+\frac{2 \sigma}{a}\right)-\frac{4}{3} \pi a^{3} \frac{2 \sigma}{a^{2}}} .
$$

The bracketed term in equation (8) describes the change in bubble size due to the gas diffusion into or out of the bubble where $R$ is the universal gas constant and $T$ is the temperature of the gas inside the bubble. The time rate of change in molar content $N_{i}$ for each gas constituent $i$ is

$$
\begin{aligned}
\frac{\partial N_{i}}{\partial t} & =4 \pi a^{2} k_{i}\left(C_{i}-\frac{P_{B, i}}{H_{i}}\right) \\
& =4 \pi a^{2} k_{i}\left(C_{i}-\frac{N_{i} R T}{4 / 3 \pi a^{3} H_{i}}\right),
\end{aligned}
$$

where $k$ is the gas transfer rate, $C$ is the aqueous concentration of the gas inside the bubble, $H$ is the Henry's law constant for the gas inside the bubble, and $P_{B}$ is the time dependent partial pressure of the gas inside the bubble. The values for $k, C, P_{B}$, and $H$ all depend on the type of gas under consideration. For the model used here, only $\mathrm{O}_{2}$ and $\mathrm{N}_{2}$ were considered (the model assumes air bubbles). A reduction in size occurs when $H C<P_{B}$, which is generally the case for air bubbles in water because of the increased gas pressure inside the bubble due to hydrostatic pressure and surface tension which can cause diffusion into what might otherwise be a saturated aqueous concentration. It is assumed here that the gas in the bubble is initially air and that the concentration of oxygen and nitrogen in the upper water column is that which describes a saturated condition at the sea surface, meaning that the aqueous concentration can be written as

$$
\begin{aligned}
C_{O 2} & =\frac{P_{o, O 2}}{H_{O 2}} \\
C_{N 2} & =\frac{P_{o, N 2}}{H_{N 2}},
\end{aligned}
$$

where $P_{o, O 2}$ and $P_{o, N 2}$ are the partial pressures of oxygen and nitrogen at sea level. The Henry's law constants (Table 1) are found from the temperature and salinity dependent solubilities given by Weiss [1970], and the corresponding concentrations are $0.2203 \mathrm{~mol} / \mathrm{m}^{3}$ for oxygen and $0.4067 \mathrm{~mol} / \mathrm{m}^{3}$ for nitrogen.

[20] The last term in the numerator of equation (8) describes the growth of the bubble owing to the change in hydrostatic pressure caused by a change in depth. The depth $z$ is defined positive downward here, and the rate of change of depth is due to the buoyant rise of the bubbles turbulent mixing in the vertical direction, and any other advection vertically in the water column. The quantities $g$ and $\rho_{w}$ are the gravitational constant and the density of water, respectively. The pressure of the gas inside the bubble, $P_{B}$, is

$$
P_{B}=P_{A}+\rho_{w} g z+\frac{2 \sigma}{a}
$$

where $P_{A}$ is the atmospheric pressure and $\sigma$ is the surface tension of the bubble.

[21] Levich [1962] gives a formula for the total mass diffusion at low Reynolds number from which the gas transfer rate can be found (see his equation (91.5) in which the concentration far from the bubble is assumed to be zero and divide by the area of the bubble),

$$
k=2 \sqrt{\frac{1}{2 \pi} \frac{D V_{b}}{a}}
$$

where $V_{b}$ is the differential velocity between the bubble and the surrounding water, which is assumed to be adequately described by the buoyant convection speed of the bubble in the model used here. The diffusion coefficients $D$ for each gas constituent are taken from Wise and Houghton [1966] and are shown in Table 1. This result by Levich is valid for clean bubbles and is used here as an upper bound on the gas transfer rate: Bubbles in seawater become "dirty" within seconds after their creation and can become stabilized for long periods of time. The manner in which surfactants and 
Table 1. Parameters Used in the Bubble Evolution Model

\begin{tabular}{lcccc}
\hline & Description & Value & Units & Reference \\
\hline $\mathrm{H}_{\mathrm{O} 2}$ & $\begin{array}{c}\text { Henry's law constant } \\
\text { for } \mathrm{O}_{2} \text { at } 20^{\circ} \mathrm{C} \text { and } 30 \mathrm{ppt} \\
\text { Henry's law constant }\end{array}$ & $9.6576 \times 10^{4}$ & $\mathrm{~Pa} / \mathrm{mol} \mathrm{m}^{-3}$ & Weiss [1970] \\
$\mathrm{H}_{\mathrm{N} 2}$ & $\begin{array}{c}\text { for } \mathrm{N}_{2} \text { at } 20^{\circ} \mathrm{C} \text { and } 30 \mathrm{ppt} \\
\text { diffusion coefficient } \\
\text { for } \mathrm{O}_{2} \text { at } 20^{\circ} \mathrm{C}\end{array}$ & $2.9433 \times 10^{5}$ & $\mathrm{~Pa} / \mathrm{mol} \mathrm{m}^{-3}$ & Weiss [1970] \\
$\mathrm{D}_{\mathrm{O} 2}$ & $\begin{array}{c}\text { diffusion coefficient } \\
\text { for } \mathrm{N}_{2} \text { at } 20^{\circ} \mathrm{C}\end{array}$ & $2.3 \times 10^{-9}$ & $\mathrm{~m}^{2} / \mathrm{s}$ & $\begin{array}{c}\text { Wise and } \\
\mathrm{D}_{\mathrm{N} 2}\end{array}$ \\
\hline
\end{tabular}

other material reduce the gas transfer rate is unknown and complicated by the potential geographic and seasonal dependencies. Clift et al. [1978] provide the following parameterization for bubbles whose skins have become immobilized (which causes differences in the boundary layer surrounding the bubble):

$$
k=0.45 g^{0.3} \nu^{0.3} a^{-0.1}\left(\frac{D}{v}\right)^{2 / 3}
$$

Here the gas transfer rate is predicted to be proportional to $D^{2 / 3}$ rather then the $D^{1 / 2}$ predicted by equation (12), although this is not a significant difference here because of the approximately equal diffusion coefficients for $\mathrm{N}_{2}$ and $\mathrm{O}_{2}$. Equations (12) and (13) also exhibit a different, albeit weak, dependence on bubble radius. Leifer and Patro [2002] suggest that in the ocean the gas transfer rate will vary between the two parameterizations. The model used in this work assumes that the gas transfer rate is proportional to equation (12) and treats it as a free parameter bounded on the upper end by the formula given by Levich. The results of the model are then matched to the measurements in order to provide some estimate of what the real gas transfer might be.

[22] Fluid dynamic forcing conditions incorporated into the model include the bubble buoyancy, a simplified model for turbulence, and the vortices described in the previous section. The turbulent field is modeled using a simple random walk, where at each time step the bubble is advected by randomly generated horizontal and vertical velocities in a manner similar to Thorpe [1984]. Standard deviations of the turbulent velocities in the well-mixed wake environment were allowed to vary between $0.5 \mathrm{~cm} / \mathrm{s}$ and $5 \mathrm{~cm} / \mathrm{s}$ for different model runs (although this is a very simple model, there was no data to support a more accurate depiction of the effects of turbulence). The parameters of the vortices used in the model are described in the previous section. The model incorporated an image vortex above each vortex in order to satisfy the surface boundary condition, and the velocities from the resulting four vortices (two real and two image vortices) were superimposed. The rise speed of bubbles due to buoyancy was calculated from the formula given by Thorpe [1982] for bubbles that are rising as solid spheres due to the presence of surfactants on the bubble surfaces,

$$
V_{b}=\frac{2}{9}\left(a^{2} g / v\right)\left[\left(y^{2}+2 y\right)^{\frac{1}{2}}-y\right],
$$

where $y$ is a shape parameter given by

$$
y=10.82 \frac{v^{2}}{g a^{3}}
$$

and $v$ is the kinematic viscosity of seawater.

[23] The bubble evolution model is initialized by seeding a semi-circular shaped region with several thousand bubbles. The bubbles are randomly placed within the wake. An initial bubble size distribution that peaks around $15 \mu \mathrm{m}$ and decays from there at approximately $a^{-4}$ is used. Although this was not measured, it is thought to be a reasonable estimate based on observations of ambient bubble populations [e.g., Vagle and Farmer, 1992; Wu, 1981] and similar modeling efforts [e.g., Thorpe et al., 1992]. Other bubble size distributions corresponding to slopes of $a^{-m}$ where $m$ took values of 3.5 and 4.5 were also tested in order to examine the sensitivity of the results to the assumed bubble size distribution.

[24] The model solves the coupled equations (8) and (9) using a fourth-order Runge-Kutta routine and runs in time steps of one tenth of a second. At each time step the bubbles are assumed to be advected by the superposition of the vortices, the turbulent motion, and their own sizedependent rise speed. If a bubble becomes smaller then $5 \mu \mathrm{m}$, it is assumed to have dissolved and is no longer tracked by the model; the same is done if a bubble reaches the surface. The model was run for $20 \mathrm{~min}$, allowing sufficient time to compare the complete lifetime of the subsurface wake expression observed with the multibeam sonar.

\section{Model Results}

[25] The model was run for four different gas transfer rates corresponding to one fifth, one fifteenth, and one forty-fifth of the clean bubble gas transfer rate, $k$. The model output was recorded every $5 \mathrm{~s}$ for up to $20 \mathrm{~min}$ until all of the bubbles had dissolved or reached the surface. In all four cases the vortices transported bubbles away from the ship's track in a similar manner to that observed in the data. Bubbles entrained in the vortices were observed over the entire vertical extent of the wake. On average, bubbles were observed to make complete circulations around the vortex 6 to 7 times. In order to compare the model results with the data, the maximum wake depth is plotted as a function of distance astern of the ship for the four different gas transfer rates as well as the data (Figure 10). The results shown in this plot are for a bubble size distribution of $a^{-4}$, and did not 


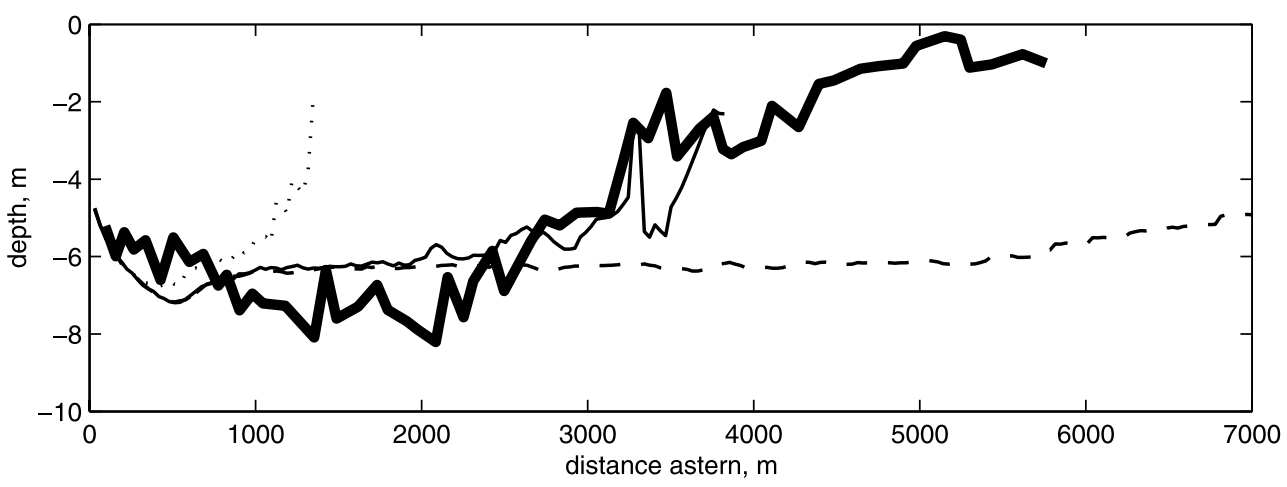

Figure 10. Maximum wake depth (averaged between port and starboard sides) for the wake observed with the multibeam sonar (thick solid line) and for four different gas transfer rates corresponding to one fifth (dotted line), one fifteenth (thin solid line), and one forty-fifth (dashed line) of the clean bubble gas transfer rate.

exhibit any significant variation from the results for the other three bubble size distributions examined. Each curve in Figure 10 corresponds to the average between port and starboard wake components. Bubbles with a gas transfer rate of one fifth of the clean bubble rate dissolved much more quickly then those observed in the multibeam data, while the bubbles with a gas transfer rate of one forty-fifth persisted for too long. In the latter case the vortices have decayed away at $5000 \mathrm{~m}$ astern of the ship (Figure 8) and the bubble cloud is slowly rising to the surface owing to buoyant convection. This indicates that it is a combination of dissolution due to gas transfer and a gradual weakening of the hydrodynamic forcing functions that leads to the reduction in wake depth observed in the data. The model output corresponding to the gas transfer rate that is one fifteenth of the clean bubble transfer rate provides the best match to the data.

[26] The model results shown in Figure 10 correspond to a turbulence model where the velocity standard deviation is $5 \mathrm{~cm} / \mathrm{s}$. Additional model runs were conducted where the turbulent velocities were up to 10 times smaller, with no significant changes in the model results for a gas transfer rate of one fifteenth of the clean bubble rate. It is possible that a much stronger turbulent intensity might influence the results here, but such a turbulent flow is not thought to be present except very close to the ship. After the passage of the ship, there are no turbulence production mechanisms present, at least in the benign weather conditions observed here. The turbulent kinetic energy (TKE) for homogenous turbulence, such as that generated by passing a uniform flow through a grid, has been observed to decay as $t^{-n}$, where $n$ is between 1.15 and 1.45 [Pope, 2000]. Although the turbulence in the ship wake is not strictly homogenous, it can be expected to decay in a similar manner. It is reasonable to assume, then, that after the first minute (or slightly less then $400 \mathrm{~m}$ astern of the ship) the TKE has decayed to $1 \%$ of its original value or less. On the basis of this estimate and the modeling results described here, shipgenerated turbulence is not expected to be a significant factor in explaining the bubble cloud persistence observed in the wake.

[27] The model prediction of the rate of change of bubble size owing to gas diffusion alone as a function of depth for a $100-\mu \mathrm{m}$-radius bubble is shown in Figure 11. This rate is approximately 5 times slower then the rate given for bubbles with an immobilized surface (equation (13)). This is generally true for larger bubbles as well, although the difference in size dependence between equations (12) and (13) has a larger effect for smaller bubbles. The ratio of the gas transfer rate for $\mathrm{N}_{2}$ for bubbles with an immobilized surface (equation (13)) to that predicted by the model (equation (12) divided by 15) is shown in Figure 12. The slower rates found here may be due to bubble stabilization by particulate matter similar to that observed in the laboratory by Johnson and Wangersky [1987].

[28] An important aspect of the model is its sensitivity to the aqueous gas concentration, which was not measured during the experiment. The dashed curves in Figure 11 correspond to gas saturations of $110 \%$ and $90 \%$. The percent error is greater near the surface, and for supersaturated water the bubbles can actually grow near the ocean surface. For bubbles at depths greater then $4 \mathrm{~m}$, a $10 \%$ error in gas saturation would correspond to a bubble decay rate

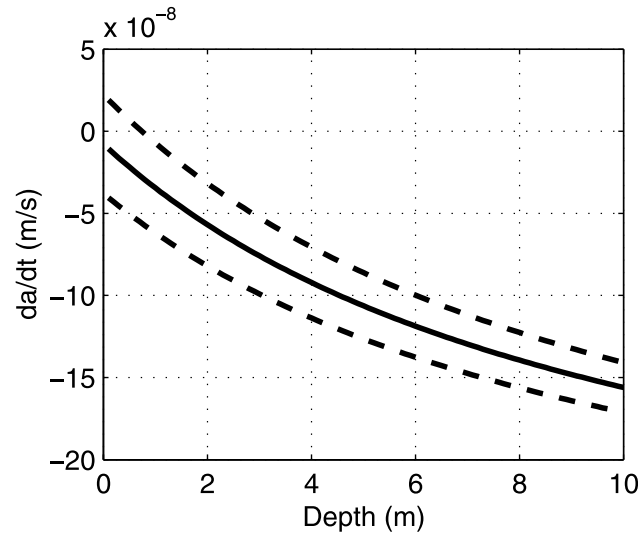

Figure 11. Rate of change of bubble size due to gas diffusion alone as a function of depth for a $70-\mu \mathrm{m}$-radius bubble. The solid line corresponds to a gas saturation of $100 \%$, and the dashed curves correspond to gas saturations of $110 \%$ and $90 \%$. 


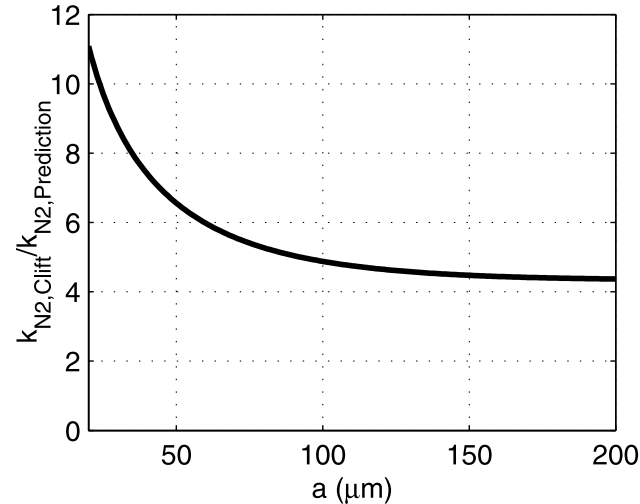

Figure 12. Ratio of the gas transfer rate predicted by Clift et al. [1978] for bubbles whose skins have become immobilized to the gas transfer rate predicted by the model results (the clean bubble gas transfer rate divided by 15 ).

error of $25 \%$ or less. Bubbles entrained in the hull-shedded vortices spend a significant amount of their time at these depths, and the gas transfer rates are expected to be valid order of magnitude estimates despite the unknown aqueous gas concentrations.

[29] Another interesting aspect of the model is the predicted spatial distribution of the bubble growth rate immediately after the ship has passed when the newly formed vortices are separated by a distance that is only slightly more then the beam of the ship. The contribution to the growth rate due to changes in hydrostatic pressure caused by the vortices and the bubble rise speed is shown in Figure 13 for a $100-\mu \mathrm{m}$ bubble. In the center of the twovortex structure, bubbles are being forced upward by the superposition of the two vortices, causing an increase in bubble size due to a decrease in hydrostatic pressure. These results indicate that a bubble size sorting process may be occurring in wakes. That is, in the center of the wake, there is a higher likelihood of finding larger bubbles, and on the edges of the wake, there is a higher likelihood of finding smaller bubbles, even if the starting distributions are the same everywhere. It is not possible to verify this with the single frequency multibeam data described here, although an indication that this may be occurring in wakes was found by Trevorrow et al. [1994]. By examining the scattering strength at two different frequencies, Trevorrow et al. observed in at least one case that the ratio of large bubbles to small bubbles was higher in the center of the wake than on the edges.

\section{Conclusions}

[30] Coupling the observed persistence of the bubble cloud created by a passing ship with a Lagrangian bubble evolution model can be an effective tool for predicting the gas transfer rate. The bubble cloud measurements discussed here were greatly aided by the wide field of view of the multibeam sonar and the benign environmental conditions. These made it possible to measure the complete evolution of the bubbles within the wake before it drifted away, a key factor in being able to extract information about the hydrodynamic forcing functions in the wake. The low wind and wave action during the experiment also minimized the injection of naturally occurring bubbles which might otherwise have been entrained within the vortices, potentially extending the persistence of the wake in time. In this case the wake appears to have been dominated by the hullshedded twin vortices. The turbulent mixing of bubbles, which is likely to be a strong factor very close to the ship, was considered to be a secondary effect because the majority of the wake analyzed was very far from the ship. Ship-generated turbulence is not expected to be responsible for maintaining the wake depth observed here.

[31] Although the hydrodynamic forcing functions for bubbles created by ship wakes or by ambient conditions are different, the mechanisms that inhibit gas dissolution from bubbles are expected to be the same. The gas transfer rate of one fifteenth of the clean bubble transfer rate defined by Levich [1962] is considered to be a good order of magnitude estimate for oceanic bubbles. It is important to note that one of the key assumptions made behind this result is that the mechanism that is inhibiting the gas transfer rate acts equally on all bubble sizes. The possibility remains, for example, that the gas transfer rate for small bubbles was one fiftieth of the clean bubble transfer rate while for larger bubbles it was one tenth. Clarification of this issue is certainly a concern, but not one that could be dealt with properly without the bubble size discrimination made possible by multifrequency acoustic measurements. A more accurate estimate is also hampered by a lack of knowledge

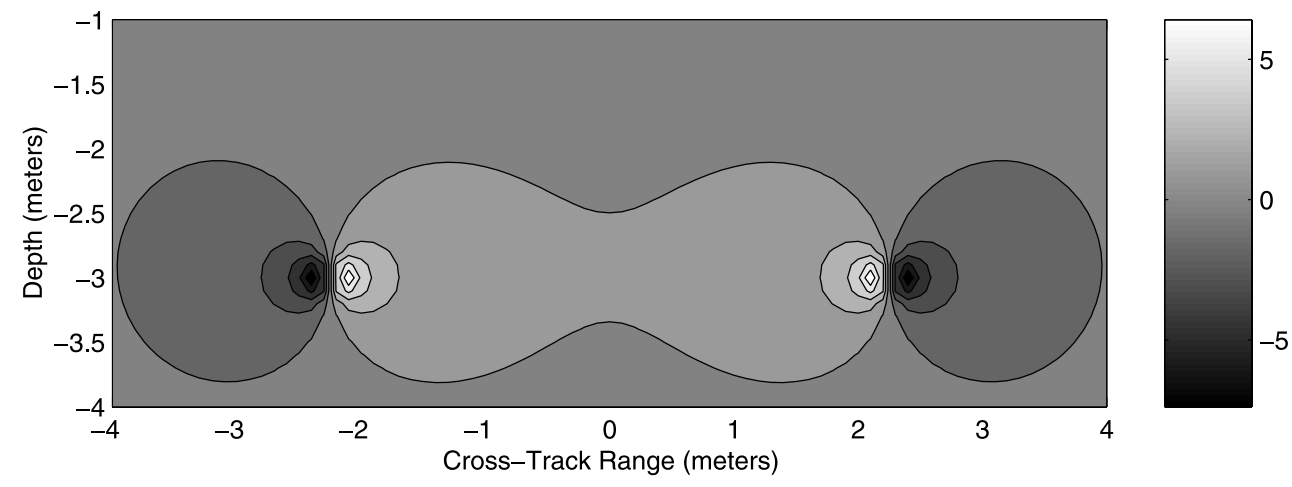

Figure 13. Spatial distribution of bubble growth, da/dt, in $\mathrm{m} / \mathrm{s}$ due exclusively to changes in hydrostatic pressure caused by the hull-shedded vortices and buoyancy for $100-\mu \mathrm{m}$-radius bubbles. 
about the aqueous concentrations of $\mathrm{O}_{2}$ and $\mathrm{N}_{2}$ present during the experiment $(100 \%$ saturation at the surface for both was assumed here). The data presented here indicate that significant bubble scavenging is occurring in order to slow the gas transfer rate, and so some significant variation in the gas transfer rate can be expected at other geographic locations and at other times of the year as surfactants and particulate populations that can act to stabilize bubbles vary.

[32] Future studies would benefit from more instrumentation in the wake. In addition to making multifrequency acoustic measurements in order to examine the evolution of different bubble sizes independently as well as measuring the aqueous concentrations of $\mathrm{O}_{2}$ and $\mathrm{N}_{2}$, it would also be useful to have more realistic descriptions of the turbulence and the hull-shedded vortices. Also, it was not possible to identify whether the bubbles are being stabilized by particulate matter or if microlayers of surfactants are the dominant mechanism here. Particulate size distributions should be measured, perhaps using optical techniques, and water samples taken to examine the nature of the surfactants. Measurements from within the wake should be compared to measurements from surrounding waters to examine the importance of surfactants released from the ship. If it can be found, a correlation between the different bubble stabilization mechanisms and the observed gas transfer rate would be very helpful when modeling the evolution of oceanic bubble plumes and their effect on air-sea gas transfer.

[33] Acknowledgments. The authors would like to thank Lee Culver and Rachel Romond of the Applied Research Laboratory and the crews of the R/V Acoustic Explorer and M/V Indianapolis for their efforts during the collection of the data presented here. The many useful comments and suggestions made by two anonymous reviewers are also greatly appreciated. This research was funded by the Office of Naval Research (code 321 US, grant N00014-04-1-0198). T. C. W. was supported by a National Defense Science and Engineering Graduate Fellowship.

\section{References}

Bischof, F., M. Sommerfeld, and F. Durst (1991), The determination of mass transfer rates from individual small bubbles, Chem. Eng. Sci., 46, 3115-3121.

Blanchard, D. C. (1989), The ejection of drops from the sea and their enrichment with bacteria and other materials: A review, Estuaries, 12 $127-137$.

Clay, C., and H. Medwin (1977), Acoustical Oceanography: Principles and Applications, 544 pp., Wiley-Interscience, New York.

Clift, R., J. Grace, and M. Weber (1978), Bubbles, Drops, and Particles, 380 pp., Elsevier, New York.

Dahl, P. (2001), Bubble clouds and their transport within the surf zone as measured with a distributed array of upward-looking sonars, J. Acoust. Soc. Am., 109, 133-142.

Farmer, D. M., C. L. McNeil, and B. D. Johnson (1993), Evidence for the importance of bubbles in increasing air-sea gas flux, Nature, 361, 620623.

Gallaudet, T. (2001), Shallow water acoustic backscatter and reverberation measurements using a $68-\mathrm{kHz}$ cylindrical array, $\mathrm{Ph}$. D. thesis, Scripps Inst. of Oceanogr., Univ. of Calif., San Diego, La Jolla.

Hyman, M., J. Rohr, J. Schoonmaker, T. Ratcliffe, B. Chadwick, K. Richter, S. Jenkins, and J. Wasyl (1995), Mixing in the wake of an aircraft carrier, paper presented at Oceans '95: Challenges of Our Global Environment, Inst. of Electr. and Electron. Eng., New York.

Johnson, B. D., and R. C. Cooke (1981), Generation of stabilized microbubbles in seawater, Science, 213, 209-211.

Johnson, B. D., and P. J. Wangersky (1987), Microbubbles: Stabilization by monolayers of adsorbed particles, J. Geophys. Res., 92, 14,641-14,647.

Kundu, P. K., and I. M. Cohen (2002), Fluid Mechanics, 2nd ed., Elsevier, New York.

Leifer, I., and R. Patro (2002), The bubble mechanism for methane transport from the shallow sea bed to the surface: A review and sensitivity study, Cont. Shelf Res., 22, 2409-2428.
Levich, V. (1962), Physicochemical Hydrodynamics, 700 pp., Prentice-Hill, Upper Saddle River, N. J.

Lyden, J. D., R. R. Hammond, D. R. Lyzenga, and R. A. Schuchman (1988), Synthetic aperture radar imaging of surface ship wakes, J. Geophys. Res., 93, 12,293-12,303.

Marmorino, G., and C. Trump (1996), Preliminary side-scan ADCP measurements across a ship's wake, J. Atmos. Oceanic Technol., 13, $507-513$.

Miner, E. W., and G. M. Griffen (1987), Bubble motions in sea water, Ocean Phys. Eng., 12(1), 25-46.

Monahan, E. C. (1986), The ocean as a source for atmospheric particles, in The Role of Air-Sea Exchange in Geochemical Cycling, edited by P. Buat-Menard, pp. 129-163, Springer, New York.

Motarjemi, M., and G. J. Jameson (1978), Mass transfer from very small bubbles-The optimum bubbles size for aeration, Chem. Eng. Sci., 33, $1415-1423$.

Nakayama, N., S. Watanabe, and S. Tsunogai (2000), Difference in $\mathrm{O}_{2}$ and $\mathrm{CO}_{2}$ gas transfer velocities in Funka Bay, Mar. Chem., 72, 115-129.

National Defense Research Committee (1946), Physics of Sound in the Sea, Division 6 Summary Tech. Rep., vol. 8, Natl. Defense Res. Comm., Washington, D. C.

Peltzer, R. D., O. M. Griffen, W. R. Barger, and J. A. Kaiser (1992), Highresolution measurements of surface-active film redistribution in ship wakes, J. Geophys. Res., 97, 5231-5252.

Pidsley, P. (1992), Acoustic scattering from surface ship wakes, paper presented at 1992 Conference, Inst. of Acoust., Hertfordshire, UK.

Pinkel, R., M. Merrifield, J. Smith, and H. Ramm (1995), Advances in Doppler sonar technology, paper presented at Fifth Working Conference on Current Measurement, Inst. of Electr. and Electron. Eng., New York.

Ponoth, S., and J. McLaughlin (1999), Numerical simulation of mass transfer for bubbles in water, Chem. Eng. Sci., 55, 1237-1255.

Pope, S. B. (2000), Turbulent Flows, 771 pp., Cambridge Univ. Press, New York.

Reed, A., R. Beck, O. Griffen, and R. Peltzer (1990), Hydrodynamics of remotely sensed surface ship wakes, Soc. Nav. Archit. Mar., Engl Transl., 98, 319-363.

Spiel, D. (1998), On the birth of film drops from bubbles bursting on seawater surfaces, J. Geophys. Res., 103, 24,907-24,918.

Thorpe, S. (1982), On the clouds of bubbles formed by breaking windwaves in deep water and their role in air-sea gas transfer, Philos. Trans. R. Soc. London, 304(1483), 155-210.

Thorpe, S. (1984), A model of the turbulent diffusion of bubbles below the sea surface, J. Phys. Oceanogr., 14, 841-854.

Thorpe, S. A., P. Bowyer, and D. K. Woolf (1992), Some factors affecting the size distribution of oceanic bubbles, J. Phys. Oceanogr., 22, 382389.

Trevorrow, M., S. Vagle, and D. Farmer (1994), Acoustical measurements of microbubbles within ship wakes, J. Acoust. Soc. Am., 95, 1922-1930.

Turner, W. R. (1961), Microbubble persistence in fresh water, J. Acoust. Soc. Am., 33, 1223-1233.

Urick, R. J. (1983), Principles of Underwater Sound, 423 pp., McGrawHill, New York.

Vagle, S., and D. M. Farmer (1992), The measurement of bubble-size distributions by acoustical backscatter, J. Atmos. Oceanic Technol., 9, $630-644$.

Vagle, S., and D. Farmer (1998), A comparison of four methods for bubble size and void fraction measurements, IEEE J. Oceanic Eng., 23, $211-$ 222 .

Wallace, D. W., and C. D. Wirick (1992), Large air-sea gas fluxes associated with breaking waves, Nature, 356, 694-696.

Weber, T. (2004), Calibration of a Reson SeaBat 8101 Multibeam Echosounder, PSU-ARL Tech. Rep. 04-010, Appl. Res. Lab., Penn. State Univ., State College.

Weiss, R. (1970), The solubility of nitrogen, oxygen, and argon in water and seawater, Deep Sea Res., 17, 721-735.

Wise, D., and G. Houghton (1966), The diffusion coefficients of ten slightly soluble gases in water at $10-60^{\circ} \mathrm{C}$, Chem. Eng. Sci., 21, 999-1010.

Woolf, D. K. (1997), Bubbles and their role in gas exchange, in The Sea Surface and Global Change, pp. 173-205, Cambridge Univ. Press, New York.

Woolf, D. K., and S. A. Thorpe (1991), Bubbles and the air-sea exchange of gases in near-saturation conditions, J. Mar. Res., 49, 435-466.

Wu, J. (1981), Bubble populations and spectra in near-surface ocean: Summary and review of field measurements, J. Geophys. Res., 86, 457-463.

D. L. Bradley, A. P. Lyons, and T. C. Weber, Applied Research Laboratory, Pennsylvania State University, State College, PA 16804, USA. (tcw141@psu.edu) 